\title{
PERFECT POWERS GENERATED BY THE TWISTED FERMAT CUBIC
}

JONATHAN REYNOLDS

\begin{abstract}
On the twisted Fermat cubic, an elliptic divisibility sequence arises as the sequence of denominators of the multiples of a single rational point. It is shown that there are finitely many perfect powers in such a sequence whose first term is greater than 1 . Moreover, if the first term is divisible by 6 and the generating point is triple another rational point then there are no perfect powers in the sequence except possibly an $l$ th power for some $l$ dividing the order of 2 in the first term.
\end{abstract}

Keywords: Elliptic divisibility sequence; perfect powers; Fermat equation.

\section{Introduction}

A divisibility sequence is a sequence

$$
W_{1}, W_{2}, W_{3}, \ldots
$$

of integers satisfying $W_{n} \mid W_{m}$ whenever $n \mid m$. The arithmetic of these has been and continues to be of great interest. Ward [41] studied a large class of recursive divisibility sequences and gave equations for points and curves from which they can be generated (see also [32]). In particular, Lucas sequences can be generated from curves of genus 0. Although Ward did not make such a distinction, sequences generated by curves of genus 1 have become exclusively known as elliptic divisibility sequences $[20,21,24,25]$ and have applications in Logic $[11,17,18]$ as well as Cryptography [38]. See [36, 37] for background on elliptic curves (genus-1 curves with a point). Let $d \in \mathbb{Z}$ be cube-free and consider the elliptic curve

$$
C: u^{3}+v^{3}=d
$$

It is sometimes said that $C$ is a twist of the Fermat cubic. The set $C(\mathbb{Q})$ forms a group under the chord and tangent method: the (projective) point $[1,-1,0]$ is

\footnotetext{
The author is supported by a Marie Curie Intra European Fellowship (PIEF-GA-2009235210)
}

2010 Mathematics Subject Classification: primary: 11G05; secondary: 11D41 
the identity and inversion is given by reflection in the line $u=v$. Suppose that $C(\mathbb{Q})$ contains a non-torsion point $P$. Then we can write, in lowest terms,

$$
m P=\left(\frac{U_{m}}{W_{m}}, \frac{V_{m}}{W_{m}}\right) .
$$

The sequence $\left(W_{m}\right)$ is a (strong) divisibility sequence (see Proposition 3.3 in [22]). Three particular questions about divisibility sequences have received much interest:

- How many terms fail to have a primitive divisor?

- How many terms are prime?

- How many terms are a perfect power?

A primitive divisor is a prime divisor which does not divide any previous term.

\subsection{Finiteness}

Bilu, Hanrot and Voutier proved that all terms in a Lucas sequence beyond the 30th have a primitive divisor [3]. Silverman showed that finitely many terms in an elliptic divisibility sequence fail to have to have a primitive divisor [34] (see also [39]). The Fibonacci and Mersenne sequences are believed to have infinitely many prime terms $[7,8]$. The latter has produced the largest primes known to date. In [9] Chudnovsky and Chudnovsky considered the likelihood that an elliptic divisibility sequence might be a source of large primes; however, $\left(W_{m}\right)$ coming from the twisted Fermat cubic has been shown to contain only finitely many prime terms [21]. Gezer and Bizim have described the squares in some periodic divisibility sequences [23]. Using modular techniques inspired by the proof of Fermat's Last Theorem, it was finally shown in [6] that the only perfect powers in the Fibonnaci sequence are 1, 8 and 144 . We will show:

Theorem 1.1. If $W_{1}>1$ then there are finitely many perfect powers in $\left(W_{m}\right)$.

The proof of Theorem 1.1 uses the divisibility properties of $\left(W_{m}\right)$ along with a modular method for cubic binary forms given in [2]. For elliptic curves in Weierstrass form similar results have been shown in [29]. In the general case, allowing for integral points, Conjecture 1.1 in [2] would give that there are finitely many perfect powers in $\left(W_{m}\right)$.

\subsection{Uniformness}

What is particularly special about sequences $\left(W_{m}\right)$ coming from twisted Fermat cubics is that they have yielded uniform results as sharp as some of their genus-0 analogues mentioned above. It has been shown that all terms of $\left(W_{m}\right)$ beyond the first have a primitive divisor [19] and, in particular, we will make use of the fact that the second term always has a primitive divisor $p_{0}>3$ (see Section 6.2 in [19]). The number of prime terms in $\left(W_{m}\right)$ is also bounded independently of $d[22]$ and, in particular, if $P$ is triple a rational point then all terms beyond the first fail to be prime (see Theorem 1.2 in [22]). Similar results can be achieved for perfect powers. Indeed: 
Theorem 1.2. Suppose that $W_{1}$ is even and at all primes greater than $3, P$ has non-singular reduction (on a minimal Weierstrass equation for $C$ ). If $W_{m}$ is an lth power for some prime $l$ then

$$
l \leqslant \max \left\{\operatorname{ord}_{2}\left(W_{1}\right),\left(1+\sqrt{p_{0}}\right)^{2}\right\},
$$

where $p_{0}>3$ is any primitive divisor of $W_{2}$. Moreover, for fixed $l>\operatorname{ord}_{2}\left(W_{1}\right)$ the number of lth powers in $\left(W_{m}\right)$ is bounded independently of $d$.

Although the conditions in Theorem 1.2 appear to depend heavily on the point, in the next theorem we exploit the fact that group $C(\mathbb{Q})$ modulo the points of nonsingular reduction has order at most 3 for a prime greater than 3 .

Theorem 1.3. Suppose that $6 \mid W_{1}$ and $P \in 3 C(\mathbb{Q})$ (or $P$ has non-singular reduction at all primes greater than 3$)$. If $W_{m}$ is an lth power for some prime $l$ then $l \mid \operatorname{ord}_{2}\left(W_{1}\right)$. In particular, if $\operatorname{ord}_{2}\left(W_{1}\right)=1$ then $\left(W_{m}\right)$ contains no perfect powers.

The conditions in Theorem 1.3 are sometimes satisfied for every rational nontorsion point on $C$. For example, we have

Corollary 1.4. The only solutions to the Diophantine equation

$$
U^{3}+V^{3}=15 W^{3 l}
$$

with $l>1$ and $\operatorname{gcd}(U, V, W)=1$ have $W=0$.

\section{Properties of elliptic divisibility sequences}

In this section the required properties of $\left(W_{m}\right)$ are collected.

Lemma 2.1. Let $p$ be a prime. For any pair $n, m \in \mathbb{N}$, if $\operatorname{ord}_{p}\left(W_{n}\right)>0$ then

$$
\operatorname{ord}_{p}\left(W_{m n}\right)=\operatorname{ord}_{p}\left(W_{n}\right)+\operatorname{ord}_{p}(m) .
$$

Proof. See equation (10) in [22].

Proposition 2.2. For all $n, m \in \mathbb{N}$,

$$
\operatorname{gcd}\left(W_{m}, W_{n}\right)=W_{\operatorname{gcd}(m, n)}
$$

In particular, for all $n, m \in \mathbb{N}, W_{n} \mid W_{n m}$.

Proof. See Proposition 3.3 in [22].

Theorem 2.3 ([19]). If $m>1$ then $W_{m}$ has a primitive divisor. 


\section{The modular approach to Diophantine equations}

For a more thorough exploration see [13] and Chapter 15 in [10]. As is conventional, in what follows all newforms shall have weight 2 with a trivial character at some level $N$ and shall be thought of as a $q$-expansion

$$
f=q+\sum_{n \geqslant 2} c_{n} q^{n}
$$

where the field $K_{f}=\mathbb{Q}\left(c_{2}, c_{3}, \cdots\right)$ is a totally real number field. The coefficients $c_{n}$ are algebraic integers and $f$ is called rational if they all belong to $\mathbb{Z}$. For a given level $N$, the number of newforms is finite. The modular symbols algorithm [12], implemented on MAGMA [4] by William Stein, shall be used to compute the newforms at a given level.

Theorem 3.1 (Modularity Theorem). Let $E / \mathbb{Q}$ be an elliptic curve of conductor $N$. Then there exists a newform $f$ of level $N$ such that $a_{p}(E)=c_{p}$ for all primes $p \nmid N$, where $c_{p}$ is pth coefficient of $f$ and $a_{p}(E)=p+1-\# E\left(\mathbb{F}_{p}\right)$.

Proof. This is due to Taylor and Wiles $[40,42]$ in the semi-stable case. The proof was completed by Breuil, Conrad, Diamond and Taylor [5].

The modularity of elliptic curves over $\mathbb{Q}$ can be seen as a converse to

Theorem 3.2 (Eichler-Shimura). Let $f$ be a rational newform of level $N$. There exists an elliptic curve $E / \mathbb{Q}$ of conductor $N$ such that $a_{p}(E)=c_{p}$ for all primes $p \nmid N$, where $c_{p}$ is the pth coefficient of $f$ and $a_{p}(E)=p+1-\# E\left(\mathbb{F}_{p}\right)$.

Proof. See Chapter 8 of [16].

Given a rational newform of level $N$, the elliptic curves of conductor $N$ associated to it via the Eichler-Shimura theorem shall be computed using MAGMA.

Proposition 3.3. Let $E / \mathbb{Q}$ be an elliptic curve with conductor $N$ and minimal discriminant $\Delta_{\min }$. Let $l$ be an odd prime and define

$$
N_{0}(E, l):=N / \prod_{\substack{p r i m e s p \\ \\ l \mid \operatorname{ord}_{p}\left(\Delta_{\min }\right)}} p .
$$

Suppose that the Galois representation

$$
\rho_{l}^{E}: \operatorname{Gal}(\overline{\mathbb{Q}} / \mathbb{Q}) \rightarrow \operatorname{Aut}(E[l])
$$

is irreducible. Then there exists a newform $f$ of level $N_{0}(E, l)$. Also there exists a prime $\mathcal{L}$ lying above $l$ in the ring of integers $\mathcal{O}_{f}$ defined by the coefficients of $f$ such that

$$
c_{p} \equiv \begin{cases}a_{p}(E) \quad \bmod \mathcal{L} & \text { if } p \nmid l N, \\ \pm(1+p) \quad \bmod \mathcal{L} & \text { if } p \| N \text { and } p \nmid l N_{0},\end{cases}
$$


where $c_{p}$ is the pth coefficient of $f$. Furthermore, if $\mathcal{O}_{f}=\mathbb{Z}$ then

$$
c_{p} \equiv \begin{cases}a_{p}(E) \bmod l & \text { if } p \nmid N, \\ \pm(1+p) \bmod l & \text { if } p \| N \text { and } p \nmid N_{0} .\end{cases}
$$

Proof. This arose from combining modularity with level-lowering results by Ribet $[30,31]$. The strengthening in the case $\mathcal{O}_{f}=\mathbb{Z}$ is due to Kraus and Oesterlé [27]. A detailed exploration is given, for example, in Chapter 2 of [13].

Remark 3.4. Let $E / \mathbb{Q}$ be an elliptic curve with conductor $N$. Note that the exponents of the primes in the factorization of $N$ are uniformly bounded (see Section 10 in Chapter IV of [35]). In particular, only primes of bad reduction divide $N$ and if $E$ has multiplicative reduction at $p$ then $p \| N$.

Corollary 3.5. Keeping the notation of Proposition 3.3, if $p$ is a prime such that $p \nmid l N_{0}$ and $p \mid N$ then

$$
l<(1+\sqrt{p})^{2\left[K_{f}: \mathbb{Q}\right]} .
$$

Proof. See Theorem 37 in [13].

Applying Proposition 3.3 to carefully constructed Frey curves has led to the solution of many Diophantine problems. The most famous of these is Fermat's Last theorem [42] but there are now constructions for other equations and we shall make use of those described below.

\subsection{A Frey curve for cubic binary forms}

Let

$$
F(x, y)=t_{0} a^{3}+t_{1}^{2} y+t_{2} x y^{2}+t_{3} y^{3} \in \mathbb{Z}[x, y]
$$

be a separable cubic binary form. In [2] a Frey curve is given for the Diophantine equation

$$
F(a, b)=d c^{l},
$$

where $\operatorname{gcd}(a, b)=1, d \in \mathbb{Z}$ is fixed and $l \geqslant 7$ is prime. Define a Frey curve $E_{a, b}$ by

$$
E_{a, b}: y^{2}=x^{3}+a_{2} x^{2}+a_{4} x+a_{6},
$$

where

$$
\begin{aligned}
& a_{2}=t_{1} a-t_{2} b, \\
& a_{4}=t_{0} t_{2} a^{2}+\left(3 t_{0} t_{3}-t_{1} t_{2}\right) a b+t_{1} t_{3} b^{2}, \\
& a_{6}=t_{0}^{2} t_{3} a^{3}-t_{0}\left(t_{2}^{2}-2 t_{1} t_{3}\right) a^{2} b+t_{3}\left(t_{1}^{2}-2 t_{0} t_{2}\right) a b^{2}-t_{0} t_{3}^{2} b^{3} .
\end{aligned}
$$

Then $E_{a, b}$ has discriminant $16 \Delta_{F} F(a, b)^{2}$. Consider the Galois representation

$$
\rho_{l}^{a, b}: \operatorname{Gal}(\overline{\mathbb{Q}} / \mathbb{Q}) \rightarrow \operatorname{Aut}\left(E_{a, b}[l]\right) .
$$


Theorem 3.6 ([2]). Let $S$ be the set of primes dividing $2 d \Delta_{F}$. There exists a constant $\alpha(d, F) \geqslant 0$ such that if $l>\alpha(d, F)$ and $c \neq \pm 1$ then:

- the representation $\rho_{l}^{a, b}$ is irreducible;

- at any prime $p \notin S$ dividing $F(a, b)$ the equation (3) is minimal, the elliptic curve $E_{a, b}$ has multiplicative reduction and $l \mid \operatorname{ord}_{p}\left(\Delta_{\min }\left(E_{a, b}\right)\right)$.

\subsection{Recipes for Diophantine equations with signature $(l, l, l)$}

The following recipe due to Kraus [28] is taken from [10]. Consider the equation

$$
A x^{l}+B y^{l}+C z^{l}=0
$$

with non-zero pairwise coprime terms and $l \geqslant 5$ prime. Setting $R=A B C$ assume that any prime $q$ satisfies $\operatorname{ord}_{q}(R)<l$. Without lost of generality also assume that $B y^{l} \equiv 0 \bmod 2$ and $A x^{l} \equiv-1 \bmod 4$. Construct the Frey curve

$$
E_{x, y}: Y^{2}=X\left(X-A x^{l}\right)\left(X+B y^{l}\right) .
$$

The conductor $N_{x, y}$ of $E_{x, y}$ is given by

$$
N_{x, y}=2^{\alpha} \operatorname{rad}_{2}(R x y z)
$$

where

$$
\alpha= \begin{cases}1, & \text { if } \operatorname{ord}_{2}(R) \geqslant 5 \text { or } \operatorname{ord}_{2}(R)=0 \\ 1, & \text { if } 1 \leqslant \operatorname{ord}_{2}(R) \leqslant 4 \text { and } y \text { is even } \\ 0, & \text { if } \operatorname{ord}_{2}(R)=4 \text { and } y \text { is odd } \\ 3, & \text { if } 2 \leqslant \operatorname{ord}_{2}(R) \leqslant 3 \text { and } y \text { is odd } \\ 5, & \text { if } \operatorname{ord}_{2}(R)=1 \text { and } y \text { is odd. }\end{cases}
$$

Theorem 3.7 (Kraus [28]). The Galois representation

$$
\rho_{l}^{x, y}: \operatorname{Gal}(\overline{\mathbb{Q}} / \mathbb{Q}) \rightarrow \operatorname{Aut}\left(E_{x, y}[l]\right)
$$

is irreducible and $N_{0}\left(E_{x, y}, l\right)$ in Proposition 3.3 is given by

$$
N_{0}=2^{\beta} \operatorname{rad}_{2}(R),
$$

where

$$
\beta= \begin{cases}1, & \text { if } \operatorname{ord}_{2}(R) \geqslant 5 \text { or } \operatorname{ord}_{2}(R)=0 \\ 0, & \text { if } \operatorname{ord}_{2}(R)=4, \\ 1, & \text { if } 1 \leqslant \operatorname{ord}_{2}(R) \leqslant 3 \text { and } y \text { is even } \\ 3, & \text { if } 2 \leqslant \operatorname{ord}_{2}(R) \leqslant 3 \text { and } y \text { is odd } \\ 5, & \text { if } \operatorname{ord}_{2}(R)=1 \text { and } y \text { is odd. }\end{cases}
$$




\section{Proof of Theorem 1.1}

Proof of Theorem 1.1. Assume that $W_{1}>1$ and $W_{m}$ is an $l$ th power for some prime $l$. Firstly we will use the Frey curve for cubic binary forms constructed in Section 3.1 and prove the existence of a prime divisor $p$ to which Corollary 3.5 can be applied, giving a bound for $l$. Let $S$ be the set of primes dividing $27 d$. By assumption, $W_{1}$ is divisible by a prime $q$. Lemma 2.1 gives that

$$
l \leqslant \operatorname{ord}_{q}\left(W_{m}\right)=\operatorname{ord}_{q}\left(W_{1}\right)+\operatorname{ord}_{q}(m) .
$$

Using Theorem 2.3 (or that there are only finitely many solutions to a Thue-Mahler equation), let $l$ be large enough so that $W_{n}$ is divisible by a prime $p \notin S$, where

$$
n=q^{l-\operatorname{ord}_{q}\left(W_{1}\right)} \text {. }
$$

Note that we can choose this lower bound for $l$ and $p$ independently of $m$. Then, using Proposition 2.2, $p \mid W_{m}$. Now construct a Frey curve $E_{U, V}$ for the Diophantine equation

$$
U_{m}^{3}+V_{m}^{3}=d W^{l}
$$

as in Section 3.1 (in our case $F(x, y)=x^{3}+y^{3}$ ) and consider the Galois representation

$$
\rho_{l}: \operatorname{Gal}(\overline{\mathbb{Q}} / \mathbb{Q}) \rightarrow \operatorname{Aut}\left(E_{U, V}[l]\right) .
$$

Using Theorem 3.6, choose $l$ larger than some constant so that $p$ divides the conductor of $E_{U, V}$ exactly once and the primes dividing $N_{0}$ in Proposition 3.3 belong to $S$. Since there are finitely many newforms of level $N_{0}$, Corollary 3.5 bounds $l$. Finally, for fixed $l$ there are finitely many solutions by Theorem 1 in [14].

\section{Proof of Theorem 1.2}

Proof of Theorem 1.2. Assume that $W_{m}$ is an $l$ th power. We will derive an $(l, l, l)$ equation (9) which does not depend on $d$ and use the Frey curve given Section 3.2. Then, similarly to the proof of Theorem 1.1, the existence of a prime divisor $p_{0}$ will be shown which bounds $l$ via Corollary 3.5. Since $2 \mid W_{1}$, by Lemma 2.1,

$$
l \leqslant \operatorname{ord}_{2}\left(W_{m}\right)=\operatorname{ord}_{2}\left(W_{1}\right)+\operatorname{ord}_{2}(m) .
$$

Assume that $l>\operatorname{ord}_{2}\left(W_{1}\right)$. Then $\operatorname{ord}_{2}(m)>0$ so $m=2 m^{\prime}$ for some $m^{\prime}$.

A Weierstrass equation for $C$ is

$$
y^{2}=x^{3}-2^{4} 3^{3} d^{2},
$$

with coordinates $x=2^{2} 3 d /(u+v)$ and $y=2^{2} 3^{2} d(u-v) /(u+v)$. Write $x(m P)=$ $A_{m} / B_{m}^{2}$ and $y(m P)=C_{m} / B_{m}^{3}$ in lowest terms.

Lemma 5.1 (see Corollary 3.2 in [22]). Let $p=2$ or 3 . then $p \mid W_{m}$ if and only if $p \nmid A_{m}$. 
The discriminant of (4) is $-2^{12} 3^{9} d^{4}$ so, since $d$ is cube free, it is minimal at any prime larger than 3 (see Remark 1.1 in Chapter VII [36]). Note that the group of points with non-singular reduction is independent of the choice of minimal Weierstrass equation. The projective equation of (4) is

$$
Y^{2} Z=X^{3}-2^{4} 3^{3} d^{2} Z^{3} .
$$

Let $p>3$ be a prime dividing $d$. By assumption, the partial derivatives

$$
\frac{\partial C}{\partial X}=-3 X^{2}, \quad \frac{\partial C}{\partial Y}=2 Y Z \quad \text { and } \quad \frac{\partial C}{\partial Z}=Y^{2}+2^{4} 3^{4} d^{2} Z^{2}
$$

do not vanish simultaneously at $P=\left[A_{1} B_{1}, C_{1}, B_{1}^{3}\right]$ over the field $\mathbb{F}_{p}$. Hence, noting that $2 \nmid A_{m}$ from Lemma 5.1 and that non-singular points form a group, we have

$$
\operatorname{gcd}\left(A_{m}^{3}, C_{m}^{2}\right) \mid 3^{3+2 \operatorname{ord}_{3}(d)}
$$

for all $m$.

The inverses of the birational transformation are given by $u=\left(2^{2} 3^{2} d+y\right) / 6 x$ and $v=\left(2^{2} 3^{2} d-y\right) / 6 x$. Thus

$$
\frac{U_{m}}{W_{m}}=\frac{2^{2} 3^{2} d B_{m}^{3}+C_{m}}{6 A_{m} B_{m}} \quad \text { and } \quad \frac{V_{m}}{W_{m}}=\frac{2^{2} 3^{2} d B_{m}^{3}-C_{m}}{6 A_{m} B_{m}} .
$$

The assumptions made restrict the cancellation which can occur in (7) and, up to cancellation, if $W_{m}$ is an $l$ th power then so is $A_{m}$. More precisely, since $W_{m}$ is an $l$ th power and $2 \mid W_{m}$, Lemma 5.1 and (6) give that $A_{m}$ is an $l$ th power multiplied by a power of 3 . Using the duplication formula,

$$
\frac{A_{m}}{B_{m}^{2}}=\frac{A_{m^{\prime}}\left(A_{m^{\prime}}^{3}+8\left(2^{4} 3^{3} d^{2}\right) B_{m^{\prime}}^{6}\right)}{4 B_{m^{\prime}}^{2}\left(A_{m^{\prime}}^{3}-2^{4} 3^{3} d^{2} B_{m^{\prime}}^{6}\right)}=\frac{A_{m^{\prime}}\left(A_{m^{\prime}}^{3}+8\left(2^{4} 3^{3} d^{2}\right) B_{m^{\prime}}^{6}\right)}{4 B_{m^{\prime}}^{2} C_{m^{\prime}}^{2}} .
$$

Again, cancellation in (8) is restricted so $A_{m^{\prime}}$ is also an $l$ power multiplied by a power of 3. Write

$$
m=2^{\operatorname{ord}_{2}(m)} n \text {. }
$$

It follows that $A_{n}=3^{e} A^{l}$,

$$
A_{n}^{3}+8\left(2^{4} 3^{3} d^{2}\right) B_{n}^{6}=3^{f} \bar{A}^{l}
$$

and $C_{n}= \pm 3^{g} C^{l}$. Combining with $C_{n}^{2}=A_{n}^{3}-2^{4} 3^{3} d^{2} B_{n}^{6}$ gives

$$
3^{f} \bar{A}^{l}+2^{3} 3^{2 g} C^{2 l}=3^{2+3 e} A^{3 l} .
$$

Note that, by dividing (9) through by an appropriate power of 3 , we can assume that 3 divides at most one of the three terms.

Let $p_{0}>3$ be a primitive divisor of $W_{2}$. Using Proposition 2.2, $p_{0} \mid W_{2 n}$ and, since $n$ is odd, $p_{0} \mid \bar{A} C$. Now follow the recipe given in Section 3.2. The conductor of the Frey curve for (9) is

$$
N_{\bar{A}, C}=2^{3} 3^{\delta} \operatorname{rad}_{3}(\bar{A} C A)
$$


and $N_{0}=2^{3} 3^{\delta}$ in Theorem 3.7, where $\delta=0$ or 1 . There is one newform

$$
f=q-q^{3}-2 q^{5}+q^{9}+4 q^{11}+\cdots
$$

of level $N_{0}=24$. Moreover, $f$ is rational. Since $p_{0} \mid N_{\bar{A}, C}$ and $p_{0} \nmid N_{0}$,

$$
l<\left(1+\sqrt{p_{0}}\right)^{2}
$$

by Corollary 3.5. Finally, for fixed $l>1$ there are finitely many solutions to (9) (see Theorem 2 in [14]) and they are independent of $d$.

\section{Proof of Theorem 1.3}

Proof of Theorem 1.3. As in the proof of Theorem 1.2, consider $x(P)=A_{P} / B_{P}^{2}$ and $y(P)=C_{P} / B_{P}^{3}$ on the Weierstrass equation

$$
y^{2}=x^{3}-2^{4} 3^{3} d^{2}
$$

for $C$. Since $P$ is triple another rational point, a prime of bad reduction greater 3 does not divide $A_{P}$ (see Section 3 in [19]). Thus the partial derivatives (5) do not vanish simultaneously at $P$ and so at all primes greater than $3, P$ has non-singular reduction on a minimal Weierstrass for $C$.

Now follow the proof of Theorem 1.2 up to (8). Factorizing over $\mathbb{Z}[\sqrt{-3}]$ gives

$$
A_{n}^{3}=C_{n}^{2}+2^{4} 3^{3} d^{2} B_{n}^{6}=\left(C_{n}+2^{2} 3 d B_{n}^{3} \sqrt{-3}\right)\left(C_{n}-2^{2} 3 d B_{n}^{3} \sqrt{-3}\right) .
$$

We have

$$
C_{n}+2^{2} 3 d B_{n}^{3} \sqrt{-3}=(-1+\sqrt{-3})^{s}(a+b \sqrt{-3})^{3} / 2^{s+3},
$$

where $s=0,1$ or 2 and $a, b$ are integers of the same parity. If $s=0$ then

$$
2^{3}\left(C_{n}+2^{2} 3 d B_{n}^{3} \sqrt{-3}\right)=a\left(a^{2}-9 b^{2}\right)+3 b\left(a^{2}-b^{2}\right) \sqrt{-3},
$$

so

$$
\begin{aligned}
2^{3} C_{n} & =a\left(a^{2}-9 b^{2}\right), \\
2^{5} d B_{n}^{3} & =b\left(a^{2}-b^{2}\right), \\
2^{2} A_{n} & =a^{2}+3 b^{2} .
\end{aligned}
$$

If $s=1$ then

$$
\begin{aligned}
2^{4} C_{n} & =-a^{3}+9 a b^{2}-9 a^{2} b+9 b^{3}, \\
2^{6} 3 d B_{n}^{3} & =a^{3}-3 a^{2} b-9 a b^{2}+3 b^{3}, \\
2^{2} A_{n} & =a^{2}+3 b^{2} .
\end{aligned}
$$


If $s=2$ then

$$
\begin{aligned}
2^{5} C_{n} & =-2 a^{3}+18 a^{2} b+18 a b^{2}-18 b^{3}, \\
2^{7} 3 d B_{n}^{3} & =-2 a^{3}-6 a^{2} b+18 a b^{2}+6 b^{3}, \\
2^{2} A_{n} & =a^{2}+3 b^{2} .
\end{aligned}
$$

By Lemma 5.1, $6 \nmid A_{n}$ so we are in the case $s=0$.

Suppose that $W_{m}$ is a square. Then, from (8), $C_{n}= \pm C^{2}, 2 B_{n}= \pm B^{2}$ and $A_{n}=A^{2}$. Since $\operatorname{gcd}(a, b) \mid 2^{2}$, one of $b$ or $a^{2}-b^{2}$ is coprime with the odd primes dividing $d$. If it is $b$ then multiplying (10) and (12) gives

$$
\pm 2^{5}(A C)^{2}=a^{5}-6 a^{3} b^{2}-27 a b^{4}
$$

and, since $b$, up to sign, is either a square or 2 multiplied by a square, dividing by $b^{5}$ gives a rational point on the hyperelliptic curve

$$
Y^{2}=X^{5}-6 X^{3}-27 X
$$

with non-zero coordinates; but computations implemented in MAGMA confirm that the Jacobian of the curve has rank 0 and, via the method of Chabauty, there are no such points. If $a^{2}-b^{2}$ is coprime with the odd primes dividing $d$ then multiplying with (12) gives a rational point on the elliptic curve

$$
\pm Y^{2}=X^{4}+2 X^{2}-3
$$

or on the elliptic curve

$$
\pm 2^{3} Y^{2}=X^{4}+2 X^{2}-3
$$

with non-zero coordinates; but there are no such points.

Suppose that $W_{m}$ is an $l$ th power for some odd prime $l$. Then, from (8), $C_{n}$, $2 B_{n}$ and $A_{n}$ are $l$ th powers. If $a$ is odd then (10) gives $a=C^{l}, a^{2}-9 b^{2}=2^{3} \bar{C}^{l}$ and

$$
C^{2 l}-2^{3} \bar{C}^{l}=9 b^{2} .
$$

If $a$ is even then $a=2 C^{l}, a^{2}-9 b^{2}=2^{2} \bar{C}^{l}$ and

$$
2^{2} C^{2 l}-2^{2} \bar{C}^{l}=9 b^{2} .
$$

Thus, Theorem 15.3.4 in [10] (due to Bennett and Skinner [1], Ivorra [26] and Siksek [33]) and Theorem 15.3.5 in [10] (due to Darmon and Merel [15]) give that $l \leqslant 5$. If $l=3$ then we have a rational point on the elliptic curve

$$
Z^{6}+X^{3}=Y^{2}
$$

this curve has rank and gives a possible solution $\bar{C}=-1, a=C= \pm 1$ and $b= \pm 1$, but, from (11), we would have $B_{n}=0$. If $l=5$ then we have a rational point on the hyper elliptic curve

$$
Y^{2}=8^{e} X^{5}+1,
$$

where $e=0$ or 1 ; but computations implemented in MAGMA confirm, via the method of Chabauty, that no such points give a required solution. 


\section{References}

[1] Michael A. Bennett and Chris M. Skinner, Ternary Diophantine equations via Galois representations and modular forms, Canad. J. Math. 56 (2004), no. 1, $23-54$.

[2] Nicolas Billerey, Formes homogènes de degré 3 et puissances p-ièmes, J. Number Theory 128 (2008), no. 5, 1272-1294.

[3] Yu. Bilu, G. Hanrot, and P. M. Voutier, Existence of primitive divisors of Lucas and Lehmer numbers, J. Reine Angew. Math. 539 (2001), 75-122, With an appendix by M. Mignotte. MR 1863855 (2002j:11027).

[4] Wieb Bosma, John Cannon, and Catherine Playoust, The Magma algebra system. I. The user language, J. Symbolic Comput. 24 (1997), no. 3-4, 235265.

[5] Christophe Breuil, Brian Conrad, Fred Diamond, and Richard Taylor, On the modularity of elliptic curves over Q: wild 3-adic exercises, J. Amer. Math. Soc. 14 (2001), no. 4, 843-939 (electronic).

[6] Yann Bugeaud, Maurice Mignotte, and Samir Siksek, Classical and modular approaches to exponential Diophantine equations. I. Fibonacci and Lucas perfect powers, Ann. of Math. (2) 163 (2006), no. 3, 969-1018.

[7] Chris Caldwell, Mersenne primes: History, theorems and lists, http:// primes.utm.edu/mersenne/index.html.

[8] Chris Caldwell, The prime pages: Fibonacci prime, http://primes.utm. edu/glossary/page $\cdot$ php?sort=FibonacciPrime.

[9] D. V. Chudnovsky and G. V. Chudnovsky, Sequences of numbers generated by addition in formal groups and new primality and factorization tests, Adv. in Appl. Math. 7 (1986), no. 4, 385-434. MR 866702 (88h:11094)

[10] Henri Cohen, Number theory. Vol. II. Analytic and modern tools, Graduate Texts in Mathematics, vol. 240, Springer, New York, 2007.

[11] Gunther Cornelissen and Karim Zahidi, Elliptic divisibility sequences and undecidable problems about rational points, J. Reine Angew. Math. 613 (2007), 1-33.

[12] J. E. Cremona, Algorithms for modular elliptic curves, Cambridge University Press, 1997.

[13] Sander R. Dahmen, Classical and modular methods applied to Diophantine equations, Ph.D. thesis, University of Utrecht, 2008, http: //igitur-archive.library.uu.nl/dissertations/2008-0820-200949/ UUindex.html.

[14] Henri Darmon and Andrew Granville, On the equations $z^{m}=F(x, y)$ and $A x^{p}+B y^{q}=C z^{r}$, Bull. London Math. Soc. 27 (1995), no. 6, 513-543.

[15] Henri Darmon and Loïc Merel, Winding quotients and some variants of Fermat's last theorem, J. Reine Angew. Math. 490 (1997), 81-100.

[16] Fred Diamond and Jerry Shurman, A first course in modular forms, Graduate Texts in Mathematics, vol. 228, Springer-Verlag, New York, 2005.

[17] Kirsten Eisenträger and Graham Everest, Descent on elliptic curves and Hilbert's tenth problem, Proc. Amer. Math. Soc. 137 (2009), no. 6, 19511959. 
[18] Kirsten Eisenträger, Graham Everest, and Alexandra Shlapentokh, Hilbert's Tenth Problem and Mazur's Conjectures in Complementary Subrings of Number Fields, http://arxiv.org/abs/1012.4878, 2010.

[19] Graham Everest, Patrick Ingram, and Shaun Stevens, Primitive divisors on twists of Fermat's cubic, LMS J. Comput. Math. 12 (2009), 54-81.

[20] Graham Everest and Helen King, Prime powers in elliptic divisibility sequences, Math. Comp. 74 (2005), no. 252, 2061-2071 (electronic).

[21] Graham Everest, Victor Miller, and Nelson Stephens, Primes generated by elliptic curves, Proc. Amer. Math. Soc. 132 (2004), no. 4, 955-963 (electronic).

[22] Graham Everest, Ouamporn Phuksuwan, and Shaun Stevens, The uniform primality conjecture for the twisted fermat cubic, http://arxiv.org/abs/ 1003.2131, 2010.

[23] Betül Gezer and Osman Bizim, Squares in elliptic divisibility sequences, Acta Arith. 144 (2010), no. 2, 125-134.

[24] Patrick Ingram, Elliptic divisibility sequences over certain curves, J. Number Theory 123 (2007), no. 2, 473-486.

[25] Patrick Ingram and Joseph H. Silverman, Uniform estimates for primitive divisors in elliptic divisibility sequences, to appear in a forthcoming memorial volume for Serge Lang, published by Springer-Verlag.

[26] Wilfrid Ivorra, Sur les équations $x^{p}+2^{\beta} y^{p}=z^{2}$ et $x^{p}+2^{\beta} y^{p}=2 z^{2}$, Acta Arith. 108 (2003), no. 4, 327-338. MR 1979902 (2004b:11036)

[27] A. Kraus and J. Oesterlé, Sur une question de B. Mazur, Math. Ann. 293 (1992), no. 2, 259-275.

[28] Alain Kraus, Majorations effectives pour l'équation de Fermat généralisée, Canad. J. Math. 49 (1997), no. 6, 1139-1161.

[29] Jonathan Reynolds, Perfect powers in elliptic divisibility sequences, http: //arxiv.org/abs/1101.2949, 2011.

[30] K. A. Ribet, On modular representations of $\mathrm{Gal}(\overline{\mathbf{Q}} / \mathbf{Q})$ arising from modular forms, Invent. Math. 100 (1990), no. 2, 431-476.

[31] Kenneth A. Ribet, Report on mod l representations of $\operatorname{Gal}(\overline{\mathbf{Q}} / \mathbf{Q})$, Motives (Seattle, WA, 1991), Proc. Sympos. Pure Math., vol. 55, Amer. Math. Soc., Providence, RI, 1994, pp. 639-676.

[32] R. Shipsey, Elliptic divisibility sequences, Ph.D. thesis, Goldsmith's College (University of London), 2000, http://homepages.gold.ac.uk/rachel/ \#PhD.

[33] Samir Siksek, On the Diophantine equation $x^{2}=y^{p}+2^{k} z^{p}$, J. Théor. Nombres Bordeaux 15 (2003), no. 3, 839-846. MR 2142239 (2005m:11049)

[34] Joseph H. Silverman, Wieferich's criterion and the abc-conjecture, J. Number Theory 30 (1988), no. 2, 226-237.

[35] Joseph H. Silverman, Advanced topics in the arithmetic of elliptic curves, Graduate Texts in Mathematics, vol. 151, Springer-Verlag, New York, 1994.

[36] Joseph H. Silverman, The arithmetic of elliptic curves, Graduate Texts in Mathematics, vol. 106, Springer, 2009.

[37] Joseph H. Silverman and John Tate, Rational points on elliptic curves, Undergraduate Texts in Mathematics, Springer-Verlag, New York, 1992. 
[38] Katherine Stange and Kristin Lauter, The elliptic curve discrete logarithm problem and equivalent hard problems for elliptic divisibility sequences, Selected Areas in Cryptography 5381 (2008), 309-327.

[39] Marco Streng, Elliptic divisibility sequences with complex multiplication, Master's thesis, Universiteit Utrecht, 2006, http://www.warwick.ac.uk/ masjap/mthesis.pdf.

[40] Richard Taylor and Andrew Wiles, Ring-theoretic properties of certain Hecke algebras, Ann. of Math. (2) 141 (1995), no. 3, 553-572.

[41] Morgan Ward, Memoir on elliptic divisibility sequences, Amer. J. Math. 70 (1948), 31-74.

[42] Andrew Wiles, Modular elliptic curves and Fermat's last theorem, Ann. of Math. (2) 141 (1995), no. 3, 443-551.

Address: Jonathan Reynolds: Mathematisch Instituut, Universiteit Utrecht, Postbus 80.010, 3508 TA Utrecht, Nederland.

E-mail: J.M.Reynolds@uu.nl

Received: 5 April 2011 